\title{
Parasitologìa veterinaria
}

\section{Diagnóstico de Neospora caninum en ganado vacuno de Venezuela \\ Sonia Almería1, Nelitza Linarez², Estluz Mata ${ }^{3}$ \\ 1 Universidad Autónoma de Barcelona, Barcelona, España \\ 2 Universidad Centroccidental "Lisandro Alvarado", Barquisimeto, Venezuela, \\ ${ }^{3}$ Instituto de Estudios Avanzados, Caracas, Venezuela}

Introducción. Neospora caninum es un protozoario, tipo coccidio, tisular e intracelular obligado, perteneciente al filo Apicomplexa, familia Sarcocystidae. Puede infectar cánidos, rumiantes y équidos (Dubey, et al., 1996). En 1984 fue reconocido por primera vez en el perro (Bjerkas, et al.). En la actualidad, es considerado una de las principales causas de abortos en el ganado vacuno en todo el mundo.

Su diagnóstico es difícil debido a la ausencia de signos clínicos en el ganado vacuno y el número bajo de parásitos en los fetos abortados. Sin embargo, puede diagnosticarse mediante técnicas que revelan la presencia del parásito, como inmunohistoquímica, inmunofluorescencia indirecta, inmunoenzimáticos y de seroaglutinación y, más directamente, por el diagnóstico molecular por PCR.

Materiales y métodos. Se muestrearon 92 animales, de los cuales, 39,1\% resultó positivo en el análisis serológico; entre ellos una vaca de 3 años de edad, que había sufrido un aborto. Se recolectaron muestras de corazón y cerebro del feto abortado. Éstas se prepararon con la tinción de hematoxilina y eosina para el estudio histopatológico. Para el diagnóstico molecular se extrajo el ADN mediante la técnica de cloroformo-metanol y con el Wizard Genomic DNA Purification Kit. El ADN se analizó por PCR, según la metodología de Liddell, et al. (1999) y Almeria et al. (2002).

Resultados. Sólo en las muestras del corazón se obtuvo la banda correspondiente a $N$. caninum; el análisis moleculardemostróla presencia del parásito que no se evidenció por técnicas histopatológicas. También se demostró que la extracción con el kit revela una mejor calidad y cantidad de ADN.

Conclusiones. Se reporta el primer caso de aborto por $N$. caninum en Venezuela, y ofrece el uso del análisis por PCR como una herramienta estandarizada para el diagnóstico bajo las condiciones de nuestro país.

\section{Estudio filogenético de Pseudoacanthocephalus lutzi (Rhinella marinus) y Acanthocephalus spp. (Dendropsophus labialis) usando citocromo oxidasa I}

Andrea Johana López-Moreno, William David Rodríguez, Luis Francisco Becerra

Grupo de Biologia Molecular, Universidad Distrital Francisco José de Caldas, Bogotá, D.C., Colombia

Introducción. Los acantocéfalos sonendoparásitos que se encuentran en la fase adulta en el intestino de vertebrados, han sido poco estudiados y su taxonomía, según los caracteres morfológicos, ha sido problemática.

El objetivo del trabajo fue realizar un estudio filogenético del gen COlen Pseudoacanthocephalus lutzi de Rhinella marinus y Acanthocephalus spp. de Dendropsophus labialis para confirmar la reasignación del género $A$. lutzi a $P$. lutzi.

Materiales y métodos. Los especímenes se recolectaron del intestino de Rhinella marinus en la vereda Providencia del municipio de Supatá (Cundinamarca), se fijaron en etanol y mediante aplanamiento usando acetocarmín, y también del Museo de la Universidad Distrital de Bogotá recolectados en el municipio de La Calera (Cundinamarca) (Dendropsophus labialis) para su respectiva identificación taxonómica.

Al material fresco se le realizó extracción del ADN, se amplifico por PCR un segmento del gen COI y, posteriormente, se secuenció. Los análisis filogenéticos se hicieron utilizando los programas Clustal X, Modeltest, PAUP y Mr. Bayes.

Resultados. Pseudoacanthocephalus lutzi y Acanthocephalus spp. presentan diferencias morfológicas en el número de ganchos de la probóscide, 14 hileras, 5-6 filas y 12 hileras, 5 filas, 4 y 6 glándulas del cemento, principalmente.

Se secuenciaron 12 especímenes que se compararon con las secuencias del mismo género y familia por medio de tres matrices de datos combinados. Se obtuvieron índices de consistencia e índices de retención cercanos a 1. Tanto los análisis de máxima parsimonia como los bayesianos de máxima verosimilitud sustentan que $P$. lutzi y Acanthocephalus spp. son monofiléticos (100\%), las especies A. dirus, A. aguillae, A. lucci 
y A. clavula son parafiléticas de $P$. lutzi.

Conclusiones. Se encontró que las especies estudiadas pertenecen a $P$. lutzi confirmando la reasignación de $A$. lutzi a $P$. lutzi y el primer registro de $P$. lutzi en $D$. labialis.

$$
\text { - } \bullet
$$

\section{Las glucoproteínas variantes de superficie de diferentes aislamientos de Trypanosoma evansi exhiben reacción cruzada con Trypanosoma vivax}

Rocío Camargo ${ }^{1,2}$, Adriana Izquier ${ }^{2,3}$, Graciela

Uzcanga $^{1,2}$, Trina Perrone ${ }^{2,4}$, José Bubis ${ }^{2}$

1 Instituto de Estudios Avanzados, Caracas, Venezuela

2 Universidad Simón Bolívar, Caracas, Venezuela

3 Universidad Central de Venezuela, Caracas, Venezuela

4 Instituto Venezolano de Investigaciones Científicas, Caracas, Venezuela

Introducción. En Venezuela, Trypanosoma evansi y Trypanosoma vivax son responsables de la tripanosomiasis animal. A diferencia de T. evansi, el cual se expande fácilmente en múridos, la expansión de T. vivax es difícil en el laboratorio. Para desarrollar métodos de diagnóstico, nos enfocamos en la identificación de antígenos de $T$. evansi que exhiben reacción cruzada con $T$. vivax. En el presente trabajo purificamos, caracterizamos bioquímicamente y evaluamos la reacción cruzada con $T$. vivax de las glucoproteínas variantes de superficie de siete aislamientos diferentes de $T$. evansi.

Materiales y métodos. Siete aislamientos de $T$. evansi, tres de ellos obtenidos de caballos, Teva1, TeGu-N/D1 y TeAp-Mantecal01; tres provenientes de burros, TeGu-Terecay323, TeGu-Terecay03 y TeGu-TerecayTrino; y uno adquirido de chigüiro, TeAp-ElFrio 01, fueron expandidos en ratas. Los parásitos purificados se homogeneizaron y se centrifugaron, y la fracción soluble resultante se procesó para cromatografía a través de una columna de Q-sefarosa conectada en serie con una columna de S-sefarosa. Las VSGs fueron purificadas en la fracción no adsorbida a las columnas y se les determinó su carácter glicoproteico, la presencia de anclajes glicosilfosfatidil inositol, sus tamaños en estado nativo, y sus puntos isoeléctricos. Posteriormente, las glucoproteínas variantes de superficie se evaluaron mediante inmunotinción con 22 sueros de animales de campo (bovinos, equinos y chigüiros) positivos y negativos para tripanosomiasis.
Resultados. Las proteínas purificadas representaron las glucoproteínas variantes de superficie porque todas eran glucoproteínas diméricas, con una masa molecular de $\sim 120 \mathrm{kDa}$, con un anclaje de glucosilfosfatidil inositol, y consistían de varias isoformas con puntos isoeléctricos de $\sim 7$. Además, las glucoproteínas variantes de superficie purificadas fueron reconocidas por los sueros de animales infectados, ya sea con $T$. evansi o $T$. vivax, lo cual indicó la existencia de reacción cruzada.

Conclusiones. Nuestros resultados demuestran que las glucoproteínas variantes de superficie de $T$. evansipueden emplearse para el diagnóstico de la tripanosomiasis animal ocasionada por $T$. evansi o T. vivax.

\section{Inmunolocalización de metaloproteasas presentes en los productos de excreción y secreción de Mammomonogamus laryngeus}

Carlos Andrés Rodríguez-Salazar, Jhon Carlos Castaño Universidad del Quindío, Armenia, Colombia

Introducción. Mammomonogamus laryngeus es un parásito que se encuentra comúnmente en el ganado, reconocido por afecciones en el tracto respiratorio de animales y raramente en infecciones humanas. El conocimiento de la infección por este parásito es mínimo pero se sabe que en el mecanismo de capacidad patógena de otros parásitos se encuentra una serie de proteasas de tipo metaloproteasas participando en los procesos de invasión, degradación de nutrientes y evasión del sistema inmunitario entre otros, que juegan un papel importante en la infección por el parásito lo que, probablemente, es de importancia también en M. laryngeus.

El objetivo del estudio fue establecer la presencia y distribución por medio de anticuerpos murinos de cuatro metaloproteasas presentes en los productos de excreción y secreción del parásito, las cuales tienen un peso molecular de 122, 108, 94,4 y $72 \mathrm{kDa}$.

Materiales y métodos. Se recolectaron 31 parásitos adultos, a cinco se les hicieron cortes histológicos mediante micrótomo, los sobrantes se mantuvieron en cultivo para obtener productos de excreción y secreción, el cual se utilizó para aislar las metaloproteasas mediante la purificación de SDS-PAGE y para realización de Western blot. Con estas metaloproteasas purificadas se inmunizaron cuatro ratones Balb/c para obtener anticuerpos 
que permitieron reconocer estas proteasas por inmunofluorescencia en cortes del parásito; con el fin de ver la especificidad de estos anticuerpos, se hizo un Western blot.

Resultados. Se determinó la presencia de una metaloproteasa de 122 kDa en el esófago y en las microvellosidades del intestino, además de otras dos de 108 y 72 kDa en los huevos, el útero, el esófago y el intestino, y una de 94,4 kDa, en las microvellosidades del intestino, los órganos reproductores y los huevos. La especificidad de los anticuerpos antimetaloproteasas se evalúo mediante Western blot.

Conclusión. Las metaloproteasas de excreción y secreción purificadas a partir de SDS-PAGE presentaron actividad inmunógena en ratones para la producción de anticuerpos, los cuales lograron reconocer las metaloproteasas del parásito.

$$
\text { - }
$$

\section{Primera caracterización molecular a nivel de serovariedad de aislamientos de Leptospira spp. obtenidos de animales y ambiente en Colombia}

Claudia Romero-Vivas ${ }^{1}$, Virginia Rodríguez ${ }^{2}$, Alfonso Calderón², Germán Arrieta², Salim Máttar², Margaret

Cuello ${ }^{1}$, Paul Levett ${ }^{3}$, Dorothy Thiry ${ }^{1}$, Andrew Falconar ${ }^{1}$

1 Grupo de Investigaciones en Enfermedades Tropicales, Departamento de Medicina, Universidad del Norte, Barranquilla, Colombia

2 Instituto de Investigaciones Biológicas del Trópico, Universidad de Córdoba, Montería, Colombia

3 Saskatchewan Health, Provincial Laboratory, Saskatchewan, Canada

Introducción. La leptospirosis es una enfermedad febril aguda causada por leptospiras patógenas, las cuales son mantenidas naturalmente en los túbulos renales de los huéspedes naturales de mantenimiento. Si bien se conocen 20 especies de Leptospira $y$ cientos de serovariedades, algunos de ellos pertenecen a diferentes especies; la determinación molecular hasta el nivel de serovariedad de aislamientos obtenidos, permite enfocar medidas de control hacia los animales portadores, así como también la inclusión de cepas bien caracterizadas en la batería de cepas para el diagnóstico local de la infección.

El objetivo del trabajo fue caracterizar molecularmente hasta el nivel de serovariedad los aislamientos de leptospiras patógenas aisladas de roedores, perros, cerdos y aguas provenientes de la Costa Caribe colombiana.

Materiales y métodos. Los perfiles moleculares de los asilamientos de Leptospira spp., provenientes de roedores (Barranquilla), perros, cerdos y aguas (Córdoba) se obtuvierons usando la técnica de electroforesis de campo pulsado, la enzima de restricción Notl, y Salmonella braenderup H9812 como estándar. El análisis de geles, dendrogramas y coeficientes Dice se realizaron usando el software BioNumerics. Los perfiles obtenidos se compararon con una librería con más de 200 serovariedades de referencia, incluyendo serovariedades patógenas, intermedias y saprofitas. Los patrones hasta con tres fragmentos de diferencia entre cepas de una misma serovariedad se consideraron genéticamente muy relacionados y, por lo tanto, pertenecientes a una misma especie [correlación Dice (CD) de 73,7 a $100 \%$ ].

Resultados Se caracterizaron cinco aislamientos como especie y serovariedad, así: aislamiento de Rattus rattus (115): L. interrogans serovar icterohaemorrhagiae $\quad(C D=100 \%)$; cerdos, aislamiento P282: L. interrogans serovar Pomona $(C D=100 \%)$ y aislamiento P 275: L. interrogans serovar Pomona (CD=80 \%); perro, C67: $L$. noguchi serovar Australis (CD=63 \%) y agua, A42: L. kirschneri serovar Pomona (CD=60\%).

Conclusiones. Este es el primer reporte de la caracterización molecular hasta nivel de serovariedad/especie, simultáneamente, de aislamientos obtenidos en Colombia, los cuales pueden ser incluidos dentro de la batería diagnóstica en los laboratorios de referencia del país.

$$
\bullet \bullet
$$

Prevalencia de Leptospira spp. patógena, circulante en una población de roedores y perros provenientes de zonas de alto riesgo para infección humana en la ciudad de BarranquillaColombia

Claudia Romero-Vivas, Margarett Cuello, Paul Levett, Andrew Falconar

Grupo de Investigaciones en Enfermedades Tropicales, Universidad del Norte, Barranquilla, Colombia

Introducción. La transmisión de la leptospirosis en Barranquillaesfavorecidaporelclimaylascondiciones socioeconómicas. Determinamos la prevalencia y serovares de Leptospira interrogans s.l. en roedoresy perros en barrios con casos de leptospirosis humana. Metodología. Se cultivaros los macerados renales de roedores y la orina de perros en medios EMJH, EMJH-5'FU y Fletcher. La seroprevalencia se estimó por microaglutinación microscópica utilizando 24 serovariedades patógenas. Se utilizó PCR para las leptospiras patógenas (lipL32) a cultivos y muestras 
biológicas y se secuenció para determinación de especie; se caracterizó la serovariedad circulante por electroforesis de campo pulsado. Resultados. Se capturaron 69 roedores: Mus musculus (49/69), Rattus rattus (16/69) y Rattus norvegicus (4/69), y 83 perros, en los años 2008-2009, en tres barrios de Barranquilla. La seroprevalencia en $R$. rattus fue de 12,5\% (IC $0-28,7 \%$ ) con las serovariedades Grippotyphosa y Jin; para M. musculus, 20,4\% (IC ${ }_{95 \%} 9,1-31,6$ $\%)$ con las serovares Grippothyphosa, Canicola, Icterohaemorrhagiae y Georgia; finalmente, para $R$. norvegicus se obtuvo el $25 \%\left(\mathrm{IC}_{95 \%} 0-67 \%\right)$ con la serovariedad Bratislava. En Canis familiaris

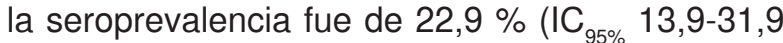
$\%)$, con las serovariedades Icterohaemorrhagiae, Lousiana y Vughia, seguidas de Hurstbridge, Bankinang, Canicola y Grippotyphosa. No hubo asociación estadísticamente significativa entre los signos y síntomas observados en los perros y la presencia o no de anticuerpos contra L. interrogans s.I. Se obtuvo un (6,3\%) aislamiento confirmándose el serovar Icterohaemorragiae en $R$. rattus $\left(\mathrm{IC}_{95 \%}\right.$ $0-18,2)$. Por PCR se detectó Leptospira patógena en $12,5 \%\left(\mathrm{IC}_{95 \%} 0-28,7 \%\right)$ de $R$. rattusy $3,7 \%\left(\mathrm{IC}_{95 \%}\right.$ $0-8,73 \%$ ) de los perros. Todos los amplicones del gen LipL32 correspondieron a L. interrogans s.s.

Conclusiones. Las serovariedades detectadas en las dos poblaciones indican el rol de otros animales, como marsupiales, cerdos y caballos, y el rol determinante de las ratas en la transmisión urbana de la leptospirosis en Barranquilla.

- $\bullet$

\section{Proliferação de células mononucleares na fasciolose crônica de ovinos experimentalmente infectados}

Eveline Albuquerque Mendes, Daniella Castanheira Bartholomeu, Vinícius Antunes Marques Ribeiro, Guilherme Rafael Gomide Pinheiro, Pedro Henrique Gazzinelli Guimarães, Ricardo Toshio Fujiwara, Walter dos Santos Lima

Departamento de Parasitologia, Instituto de Ciências Biológicas, Universidade Federal de Minas Gerais, Belo Horizonte, Minas Gerais, Brasil

A suscetibilidade a infecção por Fasciola hepatica está associada à espécie de hospedeiro e às estratégias de sobrevivência do parasito. A resposta imune celular das diferentes espécies de hospedeiros, especialmente de ovinos, é caracterizada pela proliferação transitória de células mononucleares do sangue periférico em resposta a antígenos do parasito na fase aguda da infecção.
Visando avaliar a capacidade proliferativa de células mononucleares do sangue periférico na fasciolose crônica, oito ovinos da raça Santa Inês foram experimentalmente infectados com uma média de 197,5 \pm 53,92 metacercárias de $F$. hepatica.

Aos 210 dias após a infecção, $20 \mathrm{ml}$ de sangue de cada animal, foram coletados em tubos heparinizados para separação das células mononucleares do sangue periférico . A proliferação foi avaliada pelo ensaio de MTT. As células foram incubadas por 72 horas na presença e ausência de antígeno bruto solúvel do parasito na concentração de $5 \mu \mathrm{g} / \mathrm{ml}$.

A proliferação celular foi expressa em relação à porcentagem da absorbância das culturas controles, obtida por células não tratadas, após subtração da absorbância dos poços utilizados como branco.

Os resultados mostraram que a média das absorbâncias das células estimuladas com antígeno do parasito $(0,2406 \pm 0,09387)$ não apresentaram diferença significativa na resposta proliferativa quando comparadas à média das absorbâncias obtidas das células que não receberam estímulo antigênico $(0,2217 \pm 0,09784)(p=0,6)$.

Os ovinos experimentalmente infectados por $F$. hepatica, não apresentaram uma resposta celular significativa a antígenos do parasito na fase crônica da infecção.

\section{La forma soluble de una glucoproteína variante de superficie como herramienta para el diagnóstico de la tripanosomiasis bovina}

Graciela L. Uzcanga ${ }^{1,2}$, Rocío Camargo ${ }^{1,2}$, Adriana Izquier ${ }^{2,3}$, Trina Perrone ${ }^{2,4}$, Yenis G. Pérez², José A.

$\mathrm{Noda}^{2,3}$, Francisco García ${ }^{3}$, Aurico Sousa ${ }^{3}$, Laura

P. Arias ${ }^{2}$, Ronny Chacín ${ }^{2}$, Nereida Rojas ${ }^{1,2}$, Phillipe

Büscher $^{5}$, Pedro M. Aso ${ }^{2}$, José Bubis ${ }^{2}$

1 Fundación Instituto de Estudios Avanzados, Caracas, Venezuela

2 Universidad Simón Bolívar, Caracas, Venezuela

3 Universidad Central de Venezuela, CaracasVenezuela

4 Instituto Venezolano de Investigaciones Científicas, Caracas, Venezuela

5 Institute of Tropical Medicine, Antwerp, Belgium

Introducción. El diagnóstico de la tripanosomiasis animal se ha convertido en un problema puesto que los métodos extrapolables al campo, no permiten el diagnóstico temprano de la enfermedad.

Materiales y métodos. Mediante la técnica ELISA se evaluó una glucoproteína variante de superficie 
de Trypanosoma evansi para el diagnóstico de la infección con tripanosomas y se evaluó la respuesta de anticuerpos en contra de ella a lo largo de la infección experimental de dos equinos y dos bovinos. Se estandarizó un ensayo de aglutinación en placa, empleando partículas de poliestireno carboxi-modificadas sensibilizadas con $380 \mu \mathrm{g}$ de la glucoproteína variante de superficie por cada miligramo de micropartículas.

Resultados. Los bovinos produjeron una respuesta de IgM a los diez días de la infección seguida de un incremento de la lgG, mientras los caballos generaron un pico de IgM que no pareciera madurar a una repuesta de tipo IgG. La respuesta de anticuerpos anti-glucoproteína variante de superficie fue evaluada en 43 sueros de bovinos provenientes de una zona endémica para la enfermedad en Venezuela, con una alta incidencia determinada por un método parasitológico y 60 sueros de bovinos obtenidos en una zona reportada como libre de tripanosoma en Mozambique. El 63 $\%$ de los sueros parasitológicamente positivos resultaron serológicamente positivos, pero 15 de los bovinos venezolanos parasitológicamente negativos fueron serológicamente positivos empleando ELISA para la glucoproteína variante de superficie. El $95 \%$ de los sueros provenientes de Mozambique fueron serológicamente negativos a la infección por tripanosomas. El ensayo de aglutinación fue evaluado empleando los mismos sueros usados en las pruebas de ELISA para la glucoproteína variante de superficie y se determinó que la sensibilidad del ensayo de aglutinación fue de $92 \%$ y su especificidad fue de $91 \%$.

Conclusión. La glucoproteína variante de superficie es un antígeno que puede ser empleado para el diagnóstico de la tripanosomiasis animal.

Financiamiento: proyecto G-2000001152 (FONACIT).

\section{Prevalencia de Fasciola hepatica en bovinos sacrificados en el matadero municipal de Une, Cundinamarca, en el segundo semestre de 2010}

Julio César Giraldo, Lina María Segura, Alexander

Sánchez, Juan Carlos Henao

Grupo de Investigación en Parasitología y Microbiología Tropical, Universidad Incca de Colombia, Bogotá, D.C., Colombia

Introducción. La distomatosis hepática es una enfermedad causada por Fasciola hepatica, que afecta al ganado bovino y ovino y, con menor frecuencia, al hombre como una zoonosis.
Objetivo. Determinar la prevalencia de $F$. hepatica en bovinos sacrificados en el matadero municipal de Une (Cundinamarca) en el segundo semestre del año 2010.

Metodología. Se realizó inspección post mórtem a 49 individuos de los que se obtuvo el parásito adulto por disección en hígado, contenido biliar y materia fecal durante el proceso de eviscerarlos; los adultos se lavaron con agua desionizada estéril y se almacenaron en solución tampón PBS, pH 7,2, con cloranfenicol para su preservación; el jugo biliar se centrifugó a 10.000 rpm durante 10 minutos; el precipitado se almacenó a $4^{\circ} \mathrm{C}$ para su observación microscópica; el examen coproparasitológico se realizó con la técnica Dennis.

Resultados. La prevalencia general fue de 75,0 $\%(30 / 49)$ en la cual se identificó, al menos, uno de los estadios en las muestras evaluadas. El estadio adulto se presentó en 38,78 \% (19/49) y, de éstos, $10,53 \%(2 / 19)$ en hembras y $89,47 \%$ (17/19) en machos; el estadio oval en el contenido biliar en 40,82 \% (20/49), con 10,0\% (2/20) en hembras y $90,0 \%(18 / 20)$ en machos, y el estadio oval en materia fecal en $18,38 \%(9 / 49)$, con 22,2 $\%(2 / 9)$ en hembras y $78,8 \%(7 / 9)$ en machos; la observación de estadios en las tres muestras para el mismo animal fue de $10 \%$ (3/30), la visualización de adulto en hígado y huevos en bilis en 33,3 \% (10/30), huevos en contenido biliar y materia fecal en $13,3 \%(4 / 30)$.

Conclusiones. La frecuencia de la distomatosis hepática en el matadero municipal de Une (Cundinamarca) se presenta como una de las mayores reportadas para el país. Para facilitar el control de esta zoonosis, se recomienda realizar estudios de identificación de focos ecoepidemiológicos en la región en mención.

\section{Prevalencia y factores de riesgo de Cryptosporidium spp. y Giardia spp. en terneros de ganado lechero de la zona noroccidental de la sabana de Bogotá}

Nicolás Hernández-Gallo, Jesús A. Cortés-Vecino Grupo de Parasitología Veterinaria, Facultad de Medicina Veterinaria y Zootecnia, Universidad Nacional de Colombia, Bogotá, D.C., Colombia

Introducción. La fuente más común de transmisión zoonótica por Cryptosporidium spp. y Giardia spp. está relacionada con el contacto con materia fecal - en la cual se eliminan los quistes de estos parásitos- del agua destinada a consumo humano 0 
animal. También se relaciona el contacto con heces infectadas con la leche o con algún otro producto pecuario destinado al consumo humano, en los que no se hayan tenido los cuidados sanitarios pertinentes en la producción.

El objetivo del estudio fue estimar la prevalencia de Cryptosporidium spp. y Giardia spp. en terneros de 0 a 2 meses del ganado lechero de la zona noroccidental de la sabana de Bogotá. Además, se estimaron los factores de riesgo de la producción lechera en cuanto a buenas prácticas de ganadería que puedan incurrir en la infección de personas y animales.

Métodos. Se adelantó un estudio de corte transversal, mediante la toma de materia fecal de terneros. Se observó el manejo de las buenas prácticas de ganadería en las fincas donde se obtuvieron los datos mediante un formulario de observación. Se determinó la prevalencia de punto para Giardia spp. y Cryptosporidium spp. por medio de los resultados de los exámenes coprológicos (de Ritchie para Giardia spp. y ZiehlNeelsen modificada para Cryptosporidium spp.). Se calcularon los odds ratios para establecer los factores de riesgo asociados entre estos dos géneros de protozoarios y el manejo de las buenas prácticas de ganadería.

Resultados. Se evaluaron 33 fincas dedicadas a la producción lechera, en las que se muestrearon 308 terneros. La prevalencia para Giardiaspp. fue de 37,3 \%, 115 animales positivos, y para Cryptosporidium spp. fue de 4,9\%, 15 animales positivos. Los factores de riesgo y los factores de protección tienen una fuerte asociación con la implementación de las buenas prácticas de ganadería.

Conclusión. Existe un foco de Giardia spp. y Cryptosporidium spp. en el noroccidente de la sabana de Bogotá, del cual no se tenía conocimiento previo. La prevalencia de Giardia spp. para la región estudiada, está en el límite superior de los rangos reportados para Suramérica. En el caso de Cryptosporidium spp., la situación es distinta, la prevalencia se encuentra en el límite inferior de los rangos reportados para Suramérica.

\section{- $\bullet$}

\section{Efecto nematocida in vitro del extracto de Arthrobotrys musiformis contra larvas 3 de Haemonchus contortus}

Perla María del Carmen Acevedo-Ramírez ${ }^{1}$, Rosa Ofelia Valero-Coss ${ }^{2}$, Alberto Guevara-Flores ${ }^{3}$, Héctor Quiroz-Romero', Pedro Mendoza-de Gives², Juan Luis Rendón-Gómez ${ }^{3}$
1 Facultad de Medicina Veterinaria y Zootecnia, Universidad Nacional Autónoma de México, México, D.F., México

2 Centro Nacional de Investigación Disciplinaria en Parasitología Veterinaria, Instituto Nacional de Investigaciones Forestales, Agrícolas y Pecuarias, México, D.F., México

3 Facultad de Medicina, Universidad Nacional Autónoma de México, México, D.F., México

Introducción. Haemonchus contortus es el nematodo parásito de ovinos con mayor prevalencia e importancia económica en México. En los últimos años se ha empleado el control biológico indirecto con hongos nematófagos, como Arthrobotrys musiformis.

Los objetivos fueron demostrar el efecto nematocida in vitro del extracto de Arthrobotrys musiformis contra larvas $3\left(\mathrm{~L}_{3}\right)$ de $H$. contortus y verificar la presencia de proteasas en el extracto.

Materiales y métodos. Para aislar $A$. musiformis se recolectaron muestras de distintos sustratos (tierra, raíces y heces de rumiantes) de varias regiones de México. Se determinó la capacidad depredadora de $H$. contortus contra $\mathrm{L}_{3}$. Arthrobotrys musiformis fue cultivado en medio agar papadextrosa. El micelio fue recolectado, macerado en un mortero, y después se centrifugó, se recolectó el sobrenadante (extracto crudo). Se hicieron bioensayos en pozos con $50 \mathrm{~L}_{3}$ de $\mathrm{H}$. contortus y $200 \mu \mathrm{l}$ del extracto. La actividad proteolítica se determinó utilizando caseína y gelatina como sustratos. Se hizo cromatografía de intercambio iónico.

Resultados. De 259 muestras recolectadas, se encontró A.musiformis en 6 muestras de Puebla y Veracruz. El porcentaje de captura de $L_{3}$ de $H$. contortus fue de $44 \%$. El extracto crudo causó la muerte de 83 y $89 \%$ de las $L_{3}$ de $H$. contortus, a las 24 y 48 horas, respectivamente; hubo diferencia con el control (PBS) $(p=0,000)$. El extracto presentó actividad proteolítica con actividad con caseína y gelatina. Se identificaron, al menos, dos proteasas, una de 50 kDa y otra de 36 kDa, aproximadamente.

Conclusiones. El extracto de $A$. musiformis tiene efecto nematocida in vitro contra larvas 3 de H. contortus. Dicho extracto presentó actividad proteolítica con caseína y gelatina, lo que sugiere que estas proteasas podrían ser las responsables del daño de $H$. contortus.

Nota: este trabajo será presentado en el Congreso Nacional de Parasitología, Mazatlán, Sinaloa, México, en septiembre de 2011. 